\title{
Zur relativen und absoluten Geochronologie der Reliefentwicklung an der Küste des mittleren Südwestafrika
}

\author{
Von Friedrich Wieneke \& Uwe Rust, München
}

Mit 2 Abbildungen und 1 Tabelle

\begin{abstract}
$\mathrm{Z}$ us a m menfassung. Ergebnisse quartärgeomorphologischer Arbeiten an der Namibküste werden präsentiert. Dort lassen sich zwei Meereshochstände, ein Meerestiefstand nachweisen. Der jüngere Meereshochstand ist nach 14C-Datierungen ins Innerwürm zu stellen (ca. 26000 B.P.). $\mathrm{Er}$ ist eindeutig eustatisch. Jungquartäre Vertikaltektonik im Küstenbereich ist nicht auszuschließen. Die zeitlichen Änderungen des Meeresspiegels werden mit Phasen der terrestrischen Morphogenese verknüpft. Meereshochstände korrelieren mit „trockenen“, Meerestiefstände mit „feuchten“ „Klima"-Bedingungen.

$\mathrm{S} \mathrm{u} \mathrm{m} \mathrm{m} \mathrm{a} \mathrm{ry.} \mathrm{Results} \mathrm{concerning} \mathrm{Quaternary} \mathrm{geomorphic} \mathrm{events} \mathrm{in} \mathrm{Coastal} \mathrm{Namib} \mathrm{Desert} \mathrm{are}$ presented. We found two high stands and one low stand of the sea level. We obtained radiocarbon dates of Intra-wurm age (ca. 26000 B.P.) for the younger one of the high stands. This is a eustatic high stand. Late Quaternary tectonic movements of the coastal region have to be considered. The sea level changes are linked to stages of terrestrian morphogenesis. The high stands of the sea level correspond with "arid“, the low stands with „humid“ environments.
\end{abstract}

\section{Einführung}

Im vorliegenden Aufsatz berichten wir gedrängt unter Betonung der geochronologischen Ergebnisse über Forschungen, die wir gemeinsam Januar/April 1972 an der Küste der südwestafrikanischen Namibwüste durchgeführt haben. Der Deutschen Forschungsgemeinschaft sei auch hier für ihre finanzielle Unterstützung gedankt. Eine ausführliche Darstellung der Gelände- und Laborbefunde, der verwendeten Techniken, der Kategorien der Beweisführung u. a. haben wir in RusT \& WIENEKE (1974a) vorgelegt.

Wir verfolgten das Ziel, für die Zentrale Namib die Reliefentwicklung von heute zeitlich rückwärts zu erforschen. Der Lage des Untersuchungsgebietes gemäß bedeutete dies, die Reliefentwicklung aus dem Zusammenwirken marin-litoraler, äolischer und fluvialer Morphogenese im Zeitablauf zu verstehen. Diesbezügliche Vorarbeiten lagen für unser Untersuchungsgebiet nicht vor. Vergleichbare Fragestellungen wurden von TRICART (1961) und Michel (1968) in Senegal/Mauretanien verfolgt, wo wir 1971 Gelegenheit hatten, uns im Gelände zu informieren. Da unser Ansatz geomorphologis ch ist, seien zunächst einige unseren Argumentationen zugrunde liegende Vorstellungen präzisiert.

\subsection{Zur Geomorphologie von Meeresspiegelschwankungen}

Die in Tab. 1 vorgelegte zeitliche Einstufung der Meeresspiegelschwankungen an der Namibküste geht unabhängig von den radiometrischen Daten von der Prämisse aus, daß aktuell $\mathrm{sub}$ a e r is ch vorliegende durch marine Prozesse geschaffene Formen- und Sedimentreste um so älter sind, je höher sie topographisch liegen. Die marinen Furmen und die sie aufbauenden Sedimentkörper befinden sich während der Zeitspanne ihrer Ausbildung (= Phase) in bestimmter geomorphologischer Position des jeweiligen Litorals (vgl. z. B. Ingle jr. 1966, Wieneke 1971). Gleichalte Be- 
funde können folglich in topographisch unterschiedlicher Höhe auftreten. Nicht alle Befunde für die einzelnen Phasen sind eindeutig in bestimmte Positionen des Litorals einzuordnen.

Wir haben die Formen- und Sedimentreste topographisch genau vermessen (Abb. 1, 2), bezogen auf den aktuellen STL Mile 30 (storm tide level = höchster feststellbarer Spül-

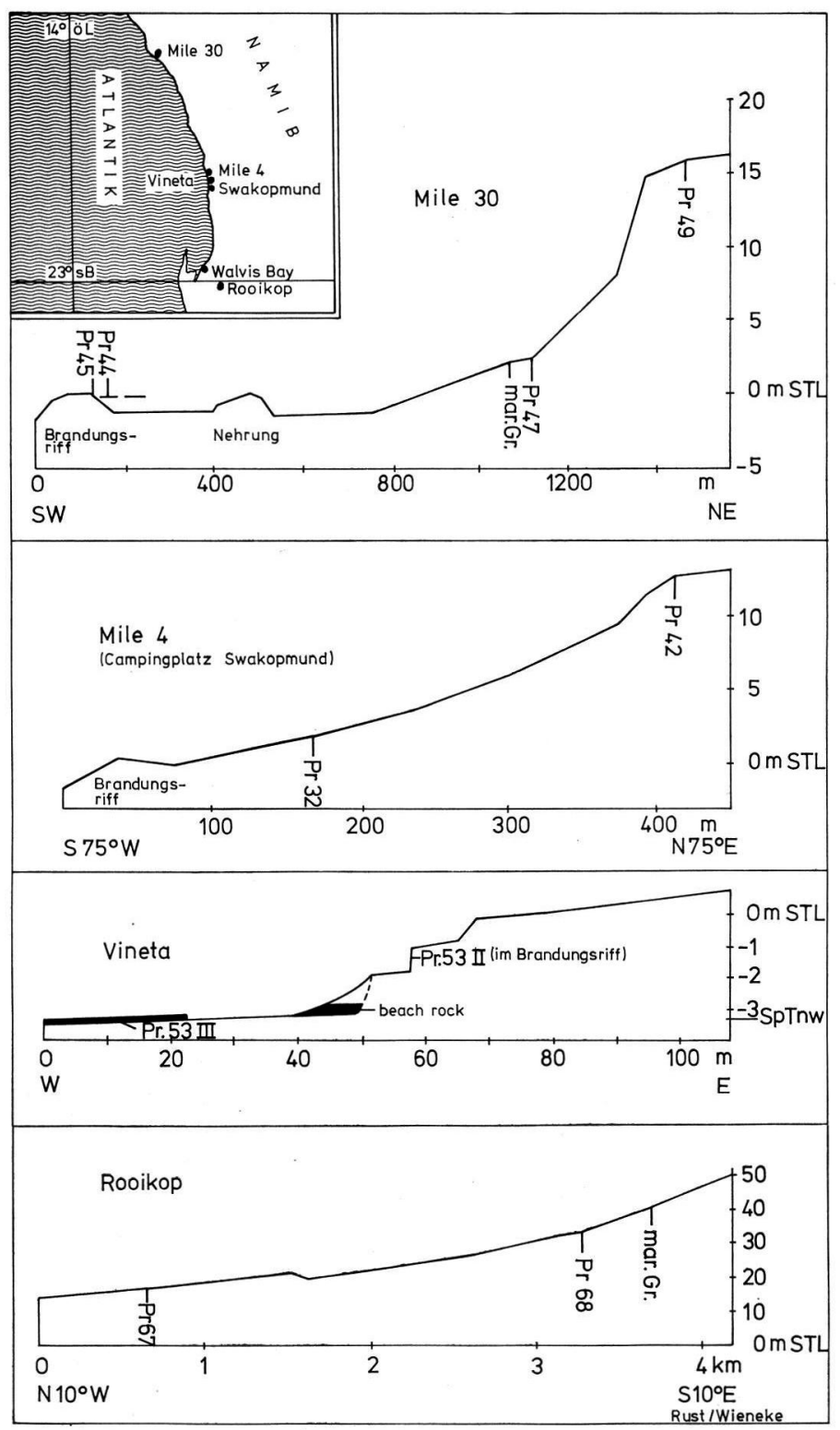

Abb. 1. Zusammengesetzte topographische Schnitte an den Lokalitäten Mile 30, Mile 4, Vineta, Rooikop (vgl. Lageskizze). Topographische Situation der gegrabenen Profile. Pr. = Einzelprobe, $\operatorname{Pr}=$ Profil, mar. Gr. = marine Grenze. - Man beachte die unterschiedlichen Vertikal- und Horizontalmaßstäbe der Schnitte (nach eigenen Vermessungen). 
saum, O m ü STL $=3.43 \mathrm{~m}$ ü Kartennull des Trigonometric Survey, Windhoek). Auf STL bezogen sind die Schwankungen des Meeresspiegels in Tab. 1 zusammengefaßt. Je nach Lage der vermessenen Befunde im jeweiligen Litoral haben dort die Schwankungsbeträge des Meeresspiegels unterschiedliche Aussagewerte (Abb. 1, 2, Tab. 1): Z. B. gibt der Betrag von $4.06 \mathrm{~m}$ zwischen Phase e (Pr. 32 VIII) und Phase b (Pr. 53 III) einen Minimalwert, da ein heute topographisch höherer Vorstrand (Pr. 32 VIII) mit einem heute tieferen nassen Strand (Pr. 53 III) verglichen wird, im Litoral ein Vorstrand stets tiefer liegt als der zugehörige nasse Strand. Andererseits ist der Betrag von $3.38 \mathrm{~m}$ zwischen Phase b (Pr. 53 III) und Phase a (STL) ein Höchstwert, da ebenfalls der nasse Strand tiefer liegt als STL, aber hier ein tieferes mit einem höheren Litoral verglichen wird. Alle Schwankungsbeträge für höher als das aktuelle Litoral vermessene Litorale sind in bezug auf STL Mindestwerte, da die vermessenen Formen- und Sedimentreste zur Zeit ihrer Ausbildung tiefer gelegen haben als der jeweils zugehörige STL der entsprechenden Phase. Unsere zentimetergenauen Angaben (z. B. $-0.29 \mathrm{~m}$ ü STL für Oberfläche von $\operatorname{Pr} 44$ in Abb. 2) haben die Qualität einer topographischen Genauigkeit; die geomorphologische Genauigkeit (Vertikalerstreckung des Litorals) umfaßt Meterdimensionen. Läßt sich die geomorphologische Position topographisch genau vermessener Punkte im Litoral angeben, so sind auch höhenmäßige Schwankungen von Litoralen unter der Schwelle der geomorphologischen Genauigkeit erkennbar (GILL 1971).

\subsection{Zur terrestrischen Geomorphologie im Trockenraum Namib}

Fluviale und äolische Formung bestimmen aktuell und in der Vergangenheit die terrestrische Morphogenese an der Namibküste, wie an einer Wüstenküste auch nicht anders zu erwarten ist. Es treten zwei unterschiedliche Arten der Talbildung (fluviale Formung) auf: Talsysteme, bei denen Nebentäler mit einem Schwemmfächer auf den Vorfluter auslaufen, sowie Talböden, auf welche keine Nebentäler ausmünden. Letzteres ist nur für die im Hochland wurzelnden allochthonen Gerinne, wie z.B. Swakop und Kuiseb, festzustellen.

Im Untersuchungsgebiet sind Gipskrusten verbreitet. Sie sind in marinen, äolischen und fluvialen Sedimenten ausgebildet (Abb. 2). Vergipsung kann hier als bodenartige Bildung in der Wüste aufgefaßt werden. Sie folgt der Sedimentbildung zeitlich nach und ändert die "morphologische Härte“ (WEBER 1958: 111-114) des Sediments. In der Zentralen Namib bestätigt sich die Ansicht von Rohdenburg (1970), daß sich an einem bestimmten Ort Formbildung und Bodenbildung zur gleichen Zeit ausschließen. Es ist aus den Geländebefunden heraus deshalb sinnvoll, mit dem Denkmodell Rohdenburgs, das zwischen den Alternativen "morphodynamische Aktivität" und „morphodynamische Stabilität" operiert, zu arbeiten. Für die fluviale Formung erweist sich weiterhin das Konzept der „Regenflächen-Spülung“ von Rust (1970) als anwendbar, weil es den flußmorphologischen Unterschied zwischen Talsystemen, die in der Namib selbst wurzeln, und Talsystemen, die im Binnenhochland wurzeln, verständlich macht und somit die beiden oben aufgezeigten Arten der Talbildung erklärt.

Wir unterscheiden zwei Möglichkeiten morphodynamischer Aktivität: „Feucht-Aktivität“ mit Bildung von Haupttälern und einmündenden Nebentälern (autochthone Talbildung), "Trocken-Aktivität“ mit Bildung von Barchanen (äolische Formung). Dazu tritt morphodynamische Stabilität in Form von „Trocken-Stabilität“ mit Gipskrustenbildung, aber gleichzeitig möglicher Eintiefung des Gerinnebettes eines im Hochland wurzelnden allochthonen Gerinnes. Wir bezeichnen diese drei fundamental voneinander verschiedenen morphodynamischen Verhältnisse als geomorphologische Milieus. Wir wollen damit andeuten, daß im Rahmen der Modellvorstellungen über die die Formbildung beeinflussenden Varianzen (BRemer 1965, s. a. BARTels 1968: 64) sich der Komplex der exogenen 
Kräfte, unter denen das Klima nur ein Glied ist, jeweils grundsätzlich unterschieden haben muß. Dabei wissen wir nicht, aus welchem Grunde und in welcher Weise (Prozesse) die Milieus sich änderten. Wir schließen nur aus bestimmten Formen- und Sedimentabfolgen auf bestimmte Milieuänderungen. Mit Hilfe unserer drei geomorphologischen Milieus läßt sich die Formenentwicklung im Trockenraum Namib hinreichend erfassen (vgl. dazu ausführlicher WIENEKE \& RUST 1973).

\section{Befunde}

An der Küste der Zentralen Namib sind zwischen etwa dem Kuiseb im S und über Mile 30 hinaus nach $N$ rezent subaerisch Reste zweier ehemaliger Litorale auffindbar. Sie sind jeweils sedimentologisch (marine Sande), geröllmorphoskopisch (Brandungsgerölle) und faunistisch (Muscheln) nachweisbar. Sie liegen in unterschiedlichen Höhen über dem heutigen Meeresspiegel (STL Mile 30) und sind eindeutig voneinander getrennt durch Formen und Sedimente terrestrischer Morphogenese. In der relativen Geochronologie unserer für die Küste der Zentralen Namib erarbeiteten Abfolge morphogenetischer Phasen (i bis $a$ in Tab. 1) sind die durch die beiden ehemaligen Litorale repräsentierten Formungsphasen mit den Buchstaben i und e bezeichnet. Die geomorphologisch-topographische Position der Formen- und Sedimentreste zueinander und zu Formen- und Sedimentresten terrestrischer Formung (Abb. 1) sowie - damit verbunden - die geomorphologisch-stratigraphische Position ausgesuchter Sediment- und Faunenproben (Abb. 2) zur Ableitung unserer Geochronologie seien an ausgewählten Lokalitäten beschrieben.

An der Lokalität Rooikop östlich Walvis ist die i-zeitliche Schorre über fast $4 \mathrm{~km}$ Horizontaldistanz als schiefe Ebene von $17.75 \mathrm{~m}$ ü STL an $\operatorname{Pr} 67$ bis $40.37 \mathrm{~m}$ ü STL (marine Grenze $=$ Geröllstrand), also über eine Vertikaldistanz von mindestens ca. $23 \mathrm{~m}$,

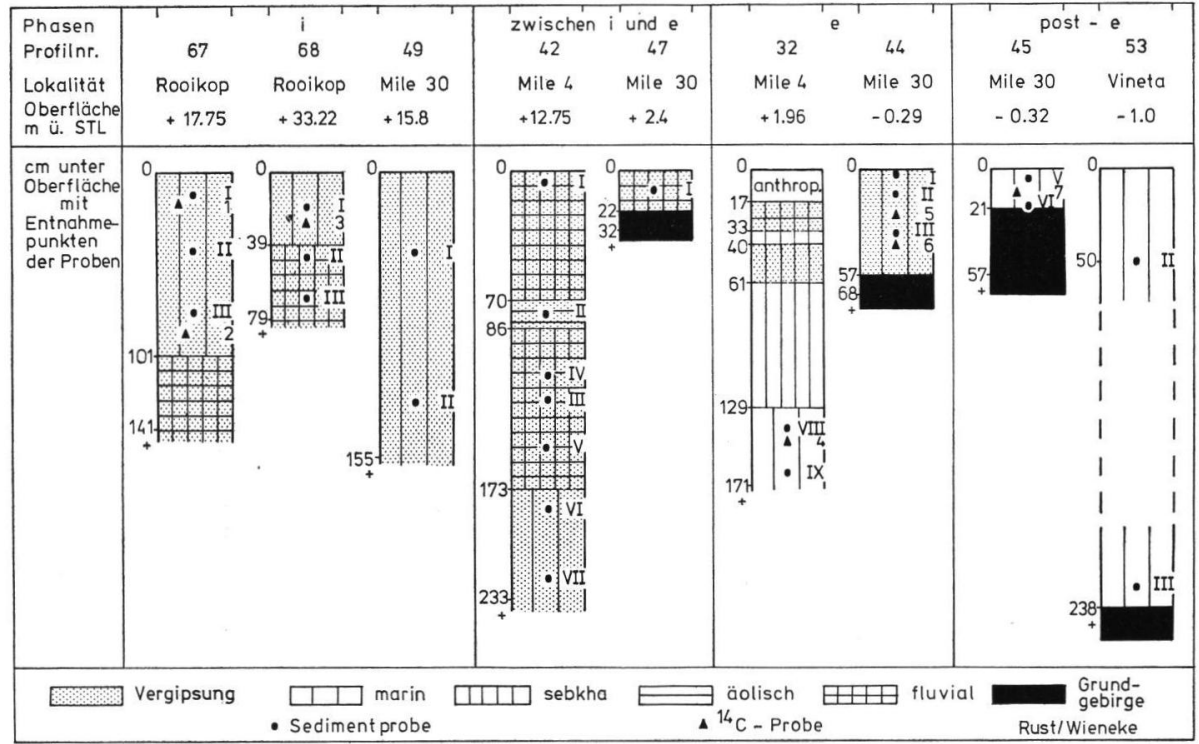

Abb. 2. Ausgewählte Profile zwischen Rooikop und Mile 30 (zur Lage vgl. Abb. 1) mit Angabe der Entnahmepunkte der Proben. Die Nummern der Sedimentproben ergeben sich aus der Profilnummer und den römischen Ziffern. Die Nummern der ${ }^{14} \mathrm{C}$-Proben lauten: $1=\mathrm{Hv}$ 5229, $2=\operatorname{Hv} 5230,3=\operatorname{Hv} 5231,4=\operatorname{Hv} 5957,5=\operatorname{Hv} 5958,6=\operatorname{Hv} 6825,7=\mathrm{Hv} 6824$. 
erhalten (Abb. 1), dokumentiert durch oberflächennahe ( $\operatorname{Pr} 67,68$ in Abb. 1, 2) und oberflächlich -flächenhaft erhaltene marine Fauna, Sande und Gerölle. Diese i-Schorre setzt sich nach $\mathrm{N}$ fort binnenwärts des Dünenstreifens zwischen Walvis Bay und Swakopmund, verzahnt sich mit der Swakopterrasse III (Rust \& WIENEKE 1974a) und ist ab Mile 4 durch fluviale Zerschneidung in Restberge aufgelöst. In $\operatorname{Pr} 67$ und $\operatorname{Pr} 68$ sind unter dem i-Marin fluviale Sedimente erhalten, die durch das i-Meer transgrediert wurden (Vergipsung prä-i der fluvialen Sedimente ?).

In Pr. 67 III liegen Muscheln als Band, meist mit der Hohlseite nach oben, flach und gut erhalten vor ( ${ }^{14} \mathrm{C}$-Probe 2), in Pr. 67 I überwiegend als zerbrochene Einzelstücke ${ }^{14} \mathrm{C}$ Probe 1), in Pr. 67 II fehlen sie. Die ${ }^{14} \mathrm{C}-$ Datierungen ergaben radiometrische Maximalalter (Pr. 67 III $=\mathrm{Hv} 5230>33485$ B.P. und Pr. I = Hv $5229>30755$ B.P.). Eine in Pr. 68 I entnommene Muschelprobe (Hv $5231=$ Pr. 3 in Abb. 2: $35950 \pm 2730 / 2170$ B.P.) ist wohl kontaminiert (Kommentar Dr. M. A. GeYH, Hannover). Daher ist der i-Hochstand altersmäßig nicht absolut zu fassen. Er ist auf jeden Fall älter, wahrscheinlich viel älter als 30000 B.P.

Bei Mile 30 (Abb. 1) ist der i-Hochstand formenmäßig faßbar durch sanft landwärts ansteigende Oberflächen von Restbergen als dort ältestes (da höchst gelegenes) Formelement. Die Formenreste lassen sich nicht mehr positionsmäßig in das ehemalige Litoral einordnen. Der erhaltene Abschnitt der i-Schorre weist eine Vertikaldistanz von 12.53 bis $20.62 \mathrm{~m}$ ü STL auf. Das die Oberflächen der Restberge konstituierende Sediment (Pr 49 in Abb. 1,2) ist sedimentologisch und geröllmorphoskopisch eindeutig marin. Es enthält nur wenig Muschelschill.

Das tiefer gelegene e-Litoral ist in Mile 30 formenmäßig dokumentiert durch ein Brandungsriff (Wallform, Schotter, marine Sande und Muscheln, Pr 45), durch eine landwärts anschließende flache ehemalige Bucht (z. B. Pr 44), in die von S eine Nehrung mit Muschelpflaster hineinreicht und die landwärts in $+2.18 \mathrm{~m}$ ü STL endet (bis dort oberflächiges Muschelvorkommen). Hier ist also das e-zeitliche Litoral von der hochenergetischen Brandungszone bis zur Schwallzone erhalten. Das e-Litoral ist von den Resten des i-Litorals durch einen von Schwemmsedimenten geringmächtig verhüllten (Pr 47) Grundgebirgshang getrennt, der durch autochthone fluviale Zerschneidung (Phase g) des i-Marin entstand und auf den das e-Meer transgredierte (keine Verzahnung fluvialer und mariner Sedimente). Dies indiziert einen Meerestiefstand zur Zeit der Phase g. ${ }^{14} \mathrm{C}$-Proben aus $\operatorname{Pr} 44$, an der landwärtigen Seite des Brandungsriffes in der ehemaligen Meeresbucht gelegen, ergaben radiometrische Alter von $25250 \pm 1150$ B.P. (Pr. $44 \mathrm{I}={ }^{14}$ C-Probe 5 in Abb. $2=$ Hv 5958) und von $26100 \pm 1835 / 1495$ B.P. (Pr. 44 II $={ }^{14} \mathrm{C}-\mathrm{Probe} 6=\mathrm{Hv} 6825$ ).

Während der Post-e-Regression wanderte das gesamte Litoral wieder meerwärts. Dadurch rückte der stationäre Wall des e-Brandungsriffs sukzessive in die strandnäheren dynamischen Zonen der surf zone und der Schwallzone, die beide niedererenergetisch sind (INGLE jr. 1966). Hierbei konnte die Geröllpackung des Walles nicht mehr abtransportiert werden, sie diente als Abtragungsschutz. Der Wall wurde schließlich als Strandwall überformt, bis er in den Bereich des trockenen Strandes und sogar oberhalb des Sturmflutniveaus rückte. Während der holozänen Transgression (Kap.3.) ist der Wall wieder in den Bereich der Schwallzone gerückt. Die ${ }^{14} \mathrm{C}$-Datierung einer Muschelprobe aus $\operatorname{Pr} 45$ ergab ein Alter von $1465 \pm 75$ B.P. ( $\mathrm{Hv} 6824={ }^{14} \mathrm{C}$-Probe 7 in Abb. 2). Post-e fand in Mile 30 noch eine geringe autochthone Zerschneidung statt (Phase c) sowie rezent Vergipsung, Regbildung und Kupstendünenbildung auf dem Wall.

Das Brandungsriff ist als Wallform durchgehend verfolgbar von Hentiesbaai im $\mathrm{N}$ bis 12 miles südlich Swakopmund. Südlich dieses Punktes haben wir es nicht mehr gefunden. Als marinlitorale Altform verknüptt es so die e-Litorale der verschiedenen Lokalitäten 
miteinander. In Mile 4 (Campingplatz von Swakopmund) ist die landwärts gelegene zugehörige surf zone stratigraphisch dokumentiert durch marine Sande, Gerölle und Muschelschill in $\operatorname{Pr} 32$ (Abb. 1, 2). Die ${ }^{14} \mathrm{C}-$ Datierung des Muschelschills aus $\operatorname{Pr} 32$ (14C-Probe 4 in Abb. 2 = Hv 5957) ergab ein Alter von $27100 \pm 1050$ B.P. Während der Post-e-Regression bildete sich in der ehemaligen surf zone eine Sebkha aus, in der auch Barchanwanderung stattfand (Rust \& Wieneke 1974a, Pr 32, Phase d). In $12.75 \mathrm{~m}$ ü STL (Profiloberfläche) sind in Pr 42 fluviale Sedimente der Phase g (= Terrasse IV des Swakop) über marinen Sanden der Phase i (= Terrasse III des Swakop) erhalten, was im Zusammenhang mit der erwähnten Verzahnung von i-Marin und Swakopterrasse III im Raum Swakopmund - Mile 4 eine Terrassenkreuzung im ehemaligen Swakopmündungsbereich dokumentiert (RUST \& WIENEKE $1974 \mathrm{a}$ ). Dieser Terrassenkörper, der einem g-zeitlichen Swakopdelta zugehört, wurde e-zeitlich durch das transgredierende Meer kliffbildend abradiert (Steilkante unter $\operatorname{Pr} 42$ in Abb. 1), was einerseits die Prä-e-Stabilisierung der Sedimente durch Vergipsung (Phase f), andererseits einen g-zeitlichen Tiefstand des Meeresspiegels (in bezug auf $\mathrm{i}$ und in bezug auf e) dokumentiert. Das e-Kliff wurde Post-e autochthon fluvial zerschnitten; die korrelaten Sedimente dieser Zerschneidung verschütteten die d-zeitliche Sebkha (fluviale Sedimente in Pr 32, Abb. 2; Phase c). Wie in Mile 30 wird die rezente Formung durch Vergipsung, Reg- und Kupstendünenbildung gekennzeichnet.

An der Lokalität Vineta (Abb. 1) wird das e-Brandungsriff abradiert. Ihm ist meerwärts, wie überall an der Namibküste, rezent ein steiler Sandstrand vorgelagert. Unter diesem Strand kommt auf Grundgebirge auflagernder beach rock zum Vorschein, der bis unter die SpTnw-Linie hinunterreicht und der rezent erodiert wird, von dem aber keine Gerölle in der Geröllpackung des Brandungsriffs enthalten sind. Der beach rock ist geochronologisch daher zwischen die Phasen e und a zu stellen. Aus seiner topographischen Position folgt, daß der beach rock einen Meerestiefstand dokumentiert, denn die Verfestigung des beach rocks findet subaerisch, also mindestens im intertidalen Bereich statt (Russell 1962). Da der Wall des Brandungsriffes post-e terrestrische und marin-litorale Formung trennte (er ist nur an der Mündung des allochthonen Swakop unterbrochen), ist eine geochronologische Einstufung der Phasen $d$ und $c$ einerseits und des Tiefstands andererseits problematisch (s. u., Tab. 1).

Aus den von ausgewählten Lokalitäten referierten Fakten ergeben sich die in Tab. 1 dargestellte Verknüpfung der marin-litoralen und terrestrischen morphogenetischen Zeitreihen, ihre relative Geochronologie (kleine Buchstaben i bis a) und die absolute Einstufung einiger der Phasen.

\section{Diskussion der Ergebnisse}

Für die Küste der Namibwüste ergeben unsere Befunde eine interessante P a ralle lit ät zwischen marin-litoralen und terrestrischen Reliefentwicklungen. Meerestiefstände entsprechen Phasen autochthoner Talbildung (feucht-aktive Phasen g und c), Meereshochstände entsprechen Phasen der Stabilisierung der Sedimente durch Vergipsung, der allochthonen Talbildung, teils der Barchanwanderung (Phasen $\mathrm{i}-\mathrm{h}, \mathrm{f}-\mathrm{e}-\mathrm{d}$ ). Vereinfacht (und geomorphologisch nicht ganz exakt) (vgl. Rust \& WIENEKE 1974a) entsprechen Meerestiefstände „feuchten “, Meereshochstände „trockenen“ „K 1 i ma" - B edingungen. Dies entspricht den Auffassungen Maarlevelds (1960) für den südafrikanischen Subkontinent. Dies ist gegenläufig im Vergleich zu den Befunden der französischen Forscher für das in vergleichbarer geographischer Lage auf der Nordhalbkugel untersuchte Westafrika (Michel 1968, TricArt 1961). 
Weiterhin haben wir $\mathrm{z}$ we i marine Terrassen höher als das aktuelle Litoral gefunden. Auf die aus der erhaltenen Schorre für Rooikop (Tab. 1) rekonstruierbare bedeutende Vertikaldistanz (ca. $23 \mathrm{~m}$ ) für e in e n Hochstand (das i-Marin) sei nochmals verwiesen. Die Zweiheit der marinen Terrassen läßt sich nur bedingt in Beziehung bringen $\mathrm{zu}$ älteren die Namibküste betreffenden Befunden. Für den Bereich zwischen Walvis Bay im S und der Mündung des Ugab im N diskutiert Davies (1973) nach Literaturangaben und nach eigenen Bereisungen einen tiefer gelegenen (very low platform) und fünf höher gelegene Strände (einschließlich holozäner Strandwälle). Die $6 \mathrm{~m}$-Terrasse anderer Autoren wurde weder von Davies noch von uns gefunden. Die $9 \mathrm{~m}$-Terrasse ist nur von Mündungen allochthoner Flüsse beschrieben und u. E. daher sicher nicht marin. Der Old Beach von DAvies entspricht nach seinen Positionsangaben unserem i-Marin, seine „very low platform" dem beach rock von Vineta. Die 18-20 m-Terrasse scheint unser g-zeitliches Swakopdelta zu sein, seine "holocene beaches“ entsprechen unserem e-Brandungsriff und der Post-e-Überformung desselben.

\begin{tabular}{|c|c|c|}
\hline Meeresspiegel & terrestrisches Relief & Phase \\
\hline- & & \\
\hline \multirow[t]{4}{*}{$\begin{array}{l}\text { (35950ะ2730I }>30755 \mathrm{BP} \\
\text { Rooikop } \\
\text { Mile } 30\end{array}$} & $\begin{array}{l}\text { allochthone Talbildung, } \\
\text { Vergipsung }\end{array}$ & i \\
\hline & $\begin{array}{l}\text { Barchane bis Mile } 4 \text {, } \\
\text { Vergipsung }\end{array}$ & h \\
\hline & $\begin{array}{l}\text { autochthone und } \\
\text { allochthone Talbildung }\end{array}$ & g \\
\hline & $\begin{array}{l}\text { im N: Vergipsung } \\
\text { im S: Barchane }\end{array}$ & $f$ \\
\hline $\begin{array}{lr} & 25250 \pm 1150 \mathrm{BP} \\
\text { Mile 30 } & 26100 \pm 1835 / 1495 \mathrm{BP} \\
\text { Mile 4 } & \\
& 27100 \pm 1050 \mathrm{BP}\end{array}$ & $\begin{array}{l}\text { allochthone Talbildung, } \\
\text { sonst wie "f" }\end{array}$ & e \\
\hline ? Vineta & $\begin{array}{l}\text { Sebkhd in Mile 4, } \\
\text { sonst wie "f" }\end{array}$ & d \\
\hline & $\begin{array}{l}\text { autochthone und allochthone } \\
\text { Talbildung im N, } \\
\text { Barchane im S }\end{array}$ & c \\
\hline Vineta & ? & $b$ \\
\hline $\begin{array}{l}\text { Mile } 30 \\
\text { STL "a" }\end{array}$ & $\begin{array}{l}\text { allochthone Talbildung, } \\
\text { Kupstendünen, Vergipsung, } \\
\text { Regbildung im N; } \\
\text { Barchane im S }\end{array}$ & $a$ \\
\hline $\begin{array}{lllllll}1 & 1 & 1 & 1 & 1 & 1 & 1 \\
40 & 30 & 25 & 20 & 15 & 10 & 5\end{array}$ & & Nieneke \\
\hline
\end{tabular}

Tabelle 1

Meeresspiegelschwankungen und Entwicklung des terrestrischen Reliefs zwischen Mile 30 und Rooikop. Für den Meeresspiegel ausgewählte Befunde (vgl. Abb. 2). Höhenangaben nach eigenen Vermessungen. Für e bis a alternative Verlaufskurven des Meeresspiegels, je nach Stellung des beach rocks von Vineta als Würm-Regressionshalt oder als holozäner Transgressionshalt. 
Neben der Tatsache, daß einige der von Davies (1973) zusammengestellten Terrassen wohl keine marin-litoralen sind, sei auf die von Davies (1971) selbst herausgestellte Problematik verwiesen, daß Höhenangaben ohne eindeutige Bezugsniveaus in einem gezeitenbeeinflußten Litoral (Handbuch 1964) schwer vergleichbar sind. Wir haben deshalb auch unsere Höhen durchweg zum storm tide level (als im Gelände überprüfbaren) Bezugsniveau vermessen und angegeben (Abb. 1, 2, Tab. 1).

Eine Zweiheit der marinen Terrassen wird von HeIne (1974) für Ostaustralien berichtet. Man könnte geneigt sein, hier eine südhemisphärische Parallele zur Namibküste zu sehen. Doch scheint uns dies zu gewagt, da in der Südhemisphäre andernorts etwa BuTzER \& Helgren (1972) in der Kapprovinz oder Herm \& Paskoff (1967), Paskoff (1972) an der chilenischen Küste mehrere marine Terrassen bzw. eine regional wechselnde Anzahl gefunden haben. So beschreibt auch Davies (1973) bei Oranjemund vier marine Terrassen, die an der Namibküste nach $\mathrm{N}$ abtauchen sollen.

Die von uns über das Verbindungsglied „Brandungsriff“ geomorphologisch abgeleitete Gleichaltrigkeit der e-Litorale bei Mile 30 und Mile 4 (Rust \& WIENEKE 1973) wurde durch die ${ }^{14} \mathrm{C}$-Datierungen radiometrisch bestätigt. Überrascht hat uns, daß der $2 \mathrm{~m}-\mathrm{Hoch}-$ stand den Datierungen zufolge kein holozäne r Hochs tand ist. Die Datierungen lassen ihn eindeutig mit ca. 26000 B.P. (Tab.1) in den Würm-KaltzeitKomplex stellen. Nach den von Tном (1973) für die Zuverlässigkeit von ${ }^{14} \mathrm{C}$-Daten mariner Befunde des Innerwürm-Hochstandes aufgestellten Kriterien können unsere Daten qualitativ als recht zuverlässig („superior?") angesehen werden. Es sei betont, daß radiometrische Gleichaltrigkeit existiert, obwohl die Mollusken aus $\operatorname{Pr} 44$ offensichtlich in situ Bänke sind (Kommentar Dr. M. A. GeYH, Hannover), diejenigen von Pr 32 in Brandungssedimenten als Schill geborgen worden sind - beide in eindeutiger geomorphologischer Position. Damit kann wohl der In n e r w ü r m-Hoch s t a nd als plausibel akzeptiert werden. Der Zeitraum dieses Hochstandes entspricht dem marinen Hochstand Ellesbo II (HILlEFors 1969) und ist gleichzeitig mit der würmzeitlichen Eisfreiheit im Inntal (Fliri 1973). Da es sich um einen eustatischen Hochstand handelt (s. u.), kann deshalb nicht ausgeschlossen werden, daß er mit dem Vereisungsgeschehen auf der Nordhalbkugel in genetischen Zusammenhang zu bringen ist.

Der beach rock von Vineta ist aufgrund seiner geomorphologisch-stratigraphischen Position zeitlich zwischen den Innerwürm-Hochstand (Phase e) und den aktuellen Meeresspiegelstand (Phase a) zu stellen. Da das Innerwürm-Brandungsriff als Form die geomorphologisch-stratigraphische Verzahnung zwischen terrestrischer und marin-litoraler Morphogenese verhindert hat, ist die Einstufung des beach rocks in die terrestrische Phasenabfolge nicht direkt möglich. Je nach seiner zeitlichen Einstufung in Phase d oder Phase b (Tab. 1) gewinnt der beach rock einen anderen Stellenwert im Rahmen der Meeresspiegelschwankungen. In Phase $\mathrm{d}$ gestellt, würde er einen Würm III-Regressionshalt, in Phase b gestellt, einen holozänen Transgressionshalt markieren.

Beide Einstufungen führen unter dem Aspekt rein eustatischer Meeresspiegelschwankungen zu kongruenten Aussagen über den Verlauf des holozänen Meeresspiegelanstiegs: Im mittleren Südwestafrika ist ke in holoz äner Hochstand höher als der aktuelle Meeresspiegelstand nachweisbar, d. h. der holozäne Meeresspiegelanstieg hätte sich „kontinuierlich“, ohne Oszillationen über das aktuelle Niveau hinaus vollzogen. Die Auffassungen von SHEPARd (1961) und ŠEgOTA (1972) würden für diese Küste zutreffen.

Wenn von der we $1 \mathrm{t}$ we it e $\mathrm{n}$ Existenz des holozänen $2 \mathrm{~m}$-Hochstandes auszugehen ist (FAIRBRIDGe 1961, Mörner 1971), für diesen aber an der Küste der Zentralen Namib jegliche Spuren fehlen, ist eine $\pm \mathrm{k}$ üsten parallele tektonische Bew e gung des Schelfes und der Litorale zu folgern. Diese Krustenänderung könnte ent- 
weder das Ansteigen des Meeresspiegels zum holozänen Hochstand und die nachfolgende Regression zum rezenten Strand jeweils kompensiert haben, so daß es nicht zu einer gesonderten Ausbildung des Litorals des holozänen Hochstandes kam, oder aber das Litoral dieses Hochstandes ist durch nachfolgende Krustensenkung in eine rezent subaquatische Position geraten. Die erstere Möglichkeit ist auf Grund der Mehrzahl der zu fordernden Bewegungen, für die es sonst im Gelände keine Anzeichen gibt, die unwahrscheinlichere, die zweite Möglichkeit ist wahrscheinlichere.

Eine holozäne Krustensenkung des Litorals würde prinzipiell eine Erniedrigung der Basisdistanz, d. h. eine Aufschüttungstendenz im Unterlauf der allochthonen Flüsse, denn nur diese sind in den trocken-stabilen Phasen der marinen Hochstände geomorphologisch aktiv, bewirken. Sie wirkt auf jeden Fall einer Regression des Meeresspiegels entgegen. Am Unterlauf des Swakop ist generell eine Einschneidungstendenz des Gerinnes festzustellen: Die jeweils jüngere Terrasse ist in die jeweils ältere eingeschnitten (RUsT \& WiEnEKe 1974a). Dies gilt für den gesamten Unterlauf bis zur heutigen Mündung. Es ist also die thalassokratisch und/oder milieumäßig gesteuerte Einschneidung des Swakop größer gewesen als eine gegenwirkende Absenkungsbewegung der küstennahen Kruste. Die (in Meterbeträgen) geringen Sprunghöhen der Swakop-Flußterrassen sind vielleicht zum Teil hierauf zurückzuführen. Dem erosionsverstärkenden Einfluß der Tektonik an den Mittelläufen der Gerinne (Swakop, Tumas, Kuiseb; Wieneke \& Rust 1973, Rust \& WIENEKE 1974b) entspricht anscheinend westlich eines \pm küstenparallelen Scharniers ein erosionsabschwächender Einfluß im Küstenbereich des mittleren Südwestafrika. Unter der Annahme, daß weltweit die Reste eines holozänen Hochstandes ca. $2 \mathrm{~m}$ über dem heutigen Meeresspiegel liegen, und aus der Tatsache, daß sie an der Küste der Zentralen Namib fehlen, wäre zu folgern, daß hier eine holozäne Absenkung der Kruste von mindestens $4 \mathrm{~m}$ im seewärtigen und im Küstenbereich stattgefunden hat.

Beide Denkalternativen - eustatischer Anstieg des Meeresspiegels nach SHEPARD und tektonische Stabilität der Namibküste oder eustatische Schwankungen nach FAIRBRIDGE und tektonische Absenkung der Küste - lassen das Innerw ürm-Litor a 1 mit Sicherheit als e ustat ischen Meeresspiegelhochstand erklärt sein. Denn es liegt mit $+2 \mathrm{~m}$ ü STL rezent höher als das rezente Litoral.

D a nks a g ung. Wir bedanken uns bei der DFG für eine Reisekostenbeihilfe zur Durchführung unserer Forschungsreise 1971/72. Herrn Prof. Dr. H. G. Gierloff-Emden (München) danken wir für seine Unterstützung bei der Planung und der Durchführung des Unternehmens, Herrn Dr. H. J. Rust (Windhoek) für die Förderung bei unseren Geländearbeiten in Südwestafrika, Herrn Dr. M. A. Geyh (Hannover) für die Radiocarbon-Datierung unserer Proben.

\section{Schriftenverzeichnis}

Bartels, D.: Zur wissenschaftstheoretischen Grundlegung einer Geographie des Menschen. - Erdk. Wissen 19, $225 \mathrm{~S}$. , Wiesbaden 1968.

Bremer, H.: Der Einfluß von Vorzeitformen auf die rezente Formung in einem Trockengebiet - Zentralaustralien. - Tag. Ber. u. Wiss. Abh. 34. Dtsch. Geographentag Heidelberg 1963, 181-196, Wiesbaden 1965.

Butzer, K. W. \& Helgren, D. M.: Late Cenozoic Evolution of the Cape Coast between Knysna and Cape St. Francis, South Africa. - Quatern. Res. 2, 143-169, New York/London 1972.

Davies, O.: Sea-Level During the Past 11000 Years (Africa). — Quaternaria 14, 195-204, Rom 1971.

- : Pleistocene Shorelines in the Western Cape and South West Africa. - Ann. Natal Mus. 21, 719-765, Pretoria 1973.

Fairbridge, W. R.: Eustatic Changes in Sea Level. - Physics and Chemistry of the Earth 4, 99-185, New York 1961. 
FLIRI, F.: Beiträge zur Geschichte der alpinen Würmvereisung: Forschungen am Bänderton von Baumkirchen (Inntal, Nordtirol). - Z. Geomorph. N. F. Suppl.-Bd. 16, 1-14, Berlin 1973.

GILl, E. D.: The Paris Symposium on World Sealevels of the Past 11000 Years. - Quaternaria 14, 1-6, Rom 1971.

Handbuch der Westküste Afrikas, II. Teil. - Hrsg. DHI, 491 S., Hamburg 1964.

Heine, K.: Bericht über die Exkursion D 3 vom 21.-30. 11. 1973 Coastal New South Wales and Southern Queensland, Australien. - In: Schönhals, E.: Der IX. Kongreß der Internationalen Union für Quartärforschung (INQUA) in Christchurch, Neuseeland, Dezember 1973, Eiszeitalter u. Gegenwart 25, 264-266, Ớhringen/Württ., 1974.

Herm, D. \& Paskoff, R. P.: Vorschlag zur Gliederung des marinen Quartärs in Nord- und MittelChile. - N. Jb. Geol. Paläont. Min., Mh 1967, 577-588, Stuttgart 1967.

Hillefors, A.: Vestsveriges glaciala historia och morfologi (The glacial history and morphology of Westsweden). - Medd. Lunds Univ. Geogr. Inst. Avh. 60, 319 S., Göteborg 1969.

IngLe jr., J. C.: The movement of beach sand. - Developm. in Sediment. 5, 221 S., Amsterdam 1966.

MaArleveld, G. C.: Über pleistozäne Ablagerungen im südlichen Afrika. - Erdk. 14, 35-46, Bonn 1960.

Michel, P.: Génèse et évolution de la vallée du Sénégal, de Bakel à l'embouchure, Afrique Occidentale. - Z. Geomorph. N. F. 12, 318-349, Berlin 1968.

Mörner, N. A.: Eustatic and climatic Oscillations. - Arct. and Alp. Res. 3, 167-171, Boulder/ Col. 1971.

Paskoff, R. P.: Les terrasses d'abrasion de la côte du Chili semi-aride. - International Geography 1972, ed. Adams, W. P. \& Helleiner, F. M., 1, 96-98, Montreal 1972.

Rohdenburg, H.: Morphodynamische Aktivitäts- und Stabilitätszeiten statt Pluvial- und Interpluvialzeiten. - Eiszeitalter u. Gegenwart 21, 81-96, Öhringen/Württ. 1970.

Russell, R. J.: Origin of beach rock. - Z. Geomorph. N. F. 6, 1-17, Berlin 1962.

Rust, U.: Beiträge zum Problem der Inselberglandschaften aus dem Mittleren Südwestafrika. Hamburger Geogr. Stud. 23, 278 S., Hamburg 1970.

Rust, U. \& Wieneke, F.: Grundzüge der quartären Reliefentwicklung der Zentralen Namib, Südwestafrika (Erste ausgewählte Ergebnisse einer Forschungsreise 1972). - J. SWA Wiss. Ges. 27, 5-28, Windhoek 1973.

- : Geomorphologie der küstennahen Zentralen Namib (Südwestafrika). - Manuskript, 138 S., München 1974a.

- : Studies on gramadulla formation in the middle part of the Kuiseb River, South West Africa. - Madoqua II 3, 5-15, Windhoek 1974b.

ŠEgOTA, T.: Radiocarbon Measurements and the Holocene and Late Würm Sealevel Rise. - Eiszeitalter u. Gegenwart 23/24, 107-115, O'hringen/Württ. 1973.

Shepard, F. P.: Sea level rise during the past 20,000 years. - Z. Geomorph. Suppl.-Bd. 3, 30-35, Berlin 1961.

Tном, B. G.: The dilemma of high interstadial sea levels during the last glaciation. - Progr. in Geogr. 5, 167-246, London 1973.

Tricart, J.: Notice explicative de la Carte Géomorphologique du delta du Sénégal. - Mém. BRGM 8, 137 S., Paris 1961.

WeBer, H.: Die Oberflächenformen des festen Landes. - Leipzig 1958.

WIENEKE, F.: Kurzfristige Umgestaltungen an der Alentejoküste nördlich Sines am Beispiel der Lagoa de Melides, Portugal (Schwallbedingter Transport an der Küste). - Münchener Geogr. Abh. 3, 151 S., München 1971.

WieneKe, F. \& Rust, U.: Klimageomorphologische Phasen in der Zentralen Namib (Südwestafrika). - Mitt. Geogr. Ges. München 58, 79-96, München 1973.

Manuskript eingeg. 17. 12. 1975.

Anschrift der Verf.: Priv.-Doz. Dr. Friedrich Wieneke und Priv.-Doz. Dr. Uwe Rust, Institut für Geographie der Universität, D-8000 München 2, Luisenstraße 37. 